\title{
Short review on solar neutrinos experiments and search for ster- ile neutrinos with solar neutrino detectors
}

\author{
Marco Pallavicini \\ ${ }^{1}$ Dipartimento di Fisica Università di Genova and Sezione INFN di Genova
}

\begin{abstract}
The spectroscopy of solar neutrinos is now entering the precision era, after a golden age which has led to the discovery of neutrino oscillations and the MSW effect. In this paper we summarise the current experimental knowledge in the field and its future perspectives, showing that solar neutrino detectors are and will remain a crucial tool for a deeper understanding of stars, neutrinos, and fundamental physics. We also show that solar neutrinos may become pivotal for the search of sterile neutrinos.
\end{abstract}

\section{Historical introduction}

The development of solar neutrino physics in recent years may be divided into two periods. The first, which lasted from the early 70s until 2001, began with the pioneering work of the radiochemical ${ }^{37} \mathrm{Cl}$ experiment at Homestake (1970-1994, [1]), grew with the study of high energy $(\mathrm{E}>6 \mathrm{MeV})$ solar neutrinos by means of the water Cherenkov detectors at Kamioka mine in Japan (KamiokaNDE 19831995 [2][3] and Super-KamiokaNDE 1996-present [4]), continued with the detection of low energy neutrinos with gallium radiochemical experiments (Gallex [5], SAGE [6]), and was concluded by the discovery of solar neutrino oscillations by SNO [7][8].

The second period started after the convincing discovery of neutrino oscillations and the beginning of the high precision era of solar physics, which has been initiated mainly by the SNO, KamLAND and Borexino experiments and which continues to develop thanks to upgrades and improvements of existing experiments and the design of future detectors.

The first period, certainly a golden age for neutrino physics, has witness the first detection of solar neutrinos at different energies, which have proved unambiguously that the Sun's energy has a nuclear origin, the development of a very refined Standard Solar Model (SSM), the recognition of a sharp discrepancy between the model itself and the observed neutrino rates (the so called Solar Neutrino Problem, SNP), and the triumphal discovery of neutrino flavour oscillations as the sole possible explanation of that discrepancy.

Although solar neutrino oscillations were, since the very beginning, a possible explanation of the SNP, the favour increased only when a clear evidence of oscillations was found in 1998 by means of atmospheric neutrinos [9].

The observed deficit is explained by the fact that most solar neutrino experiments are either insensitive to muon or tau neutrinos, or have a suppressed cross section. The fact that the deficit is energy dependent requires the so called MSW effect [10][11][12][13], the resonant enhancement of flavour conversion due to the coherent interaction of neutrinos in the solar medium. However, it was 
the SNO experiment that closed the story. The use of heavy water made the experiment able to measure separately the electron neutrino and the total neutrino flux at Earth, proving without ambiguities that the total neutrino flux at is the same as predicted by the SSM and that the electron neutrino flux is reduced by oscillations. Later measurements with enhanced neutron detection capability, first by dissolving salt in water and later with ${ }^{3} \mathrm{He}$ detectors, have confirmed the result. The oscillation picture was confirmed soon by the KamLAND reactor experiment [14].

The second period, which started after the discovery of neutrino oscillations, is continuing nowadays. Although main goal of solar neutrino physics has slightly changed, the focus is still both on fundamental neutrino physics and on solar science. In this paper we summarise the results obtained since 2001 on solar neutrinos by many different experiments, and particularly by Borexino at the Laboratory Nazionali del Gran Sasso. At the time of writing, the running solar neutrino experiments are Borexino, Sage, Super-kamiokaNDE and KamLAND. The SNO+ collaboration is upgrading the detector to start a new solar neutrino phase with liquid scintillator instead of heavy water. Other experiments which are under discussion include solar neutrino programs. For a recent review of solar neutrino physics see e.g. [15].

\section{Solar physics today}

For thirty years solar neutrino physics was driven by the solar neutrino problem, which was finally understood in terms of the Pontecorvo-Maki-Nakagawa-Sakata (PMNS) neutrino flavour mixing matrix, the analogous of the CKM matrix in the quark sector. Now that neutrino oscillations have been established and all mixing angles, including the relatively small $\theta_{13}$, have been measured, precise spectroscopy of the full spectrum of solar neutrinos is possible.

This fact offers two important scientific opportunities: on one hand, with weak interaction uncertainties removed, solar neutrinos can be used to probe possible limitations of the Standard Solar Model, such as, in particular, the uncertainties in the metal content (metallicity problem), and the effect of rotation, mixing, or magnetic fields. Future measurements at higher precision with respect to the one currently available, might also allow to compare the neutrino luminosity with the photon one, and study the thermal equilibrium of the Sun in a unique way. On the other hand, the neutrinos coming from the Sun remain important to fundamental physics: the spectral shapes and fluxes of the various sources are known rather precisely, offering the possibility to study neutrino properties such as, e.g. neutrino magnetic moments, the existence of non-standard neutrino-electron interactions, or the existence of sterile neutrinos.

Some of these measurement are possible within a relatively short time scale, others are more for the future. In particular, a more precise measurement of ${ }^{7} \mathrm{Be}$, pep, and ${ }^{8} \mathrm{~B}$ neutrinos might constraint the $\mathrm{P}_{e e}$ to a level which might be sufficient to probe the MSW effect and search for new physics. A first direct measurement of pp neutrinos might be behind the corner (Borexino), although probably not with sufficient precision such as it would be needed to probe the solar luminosity constraint.

The metallicity problem might be solved by a precise direct measurement of CNO neutrinos. This is certainly a very difficult task which might be faced wither by Borexino or by the SNO+ experiment. A future detector such as LENS might measure all neutrino fluxes neatly and with small background.

The paper is structured as follows; section 2 summarise the many important goals of solar neutrino physics today; section 3 is devoted to the results obtained on ${ }^{8} \mathrm{~B}$ neutrinos by Borexino, by KamLAND, and by the SNO and Super-KamiokaNDE experiments, which have made continuos improvement on their detectors and on their analysis methods, reducing the energy threshold and improving the statistics ; section 4 is devoted to a summary of the results obtained on sub-MeV neutrinos by Borexino, definitely the main solar neutrino results obtained recently; section 5 outlines the main 


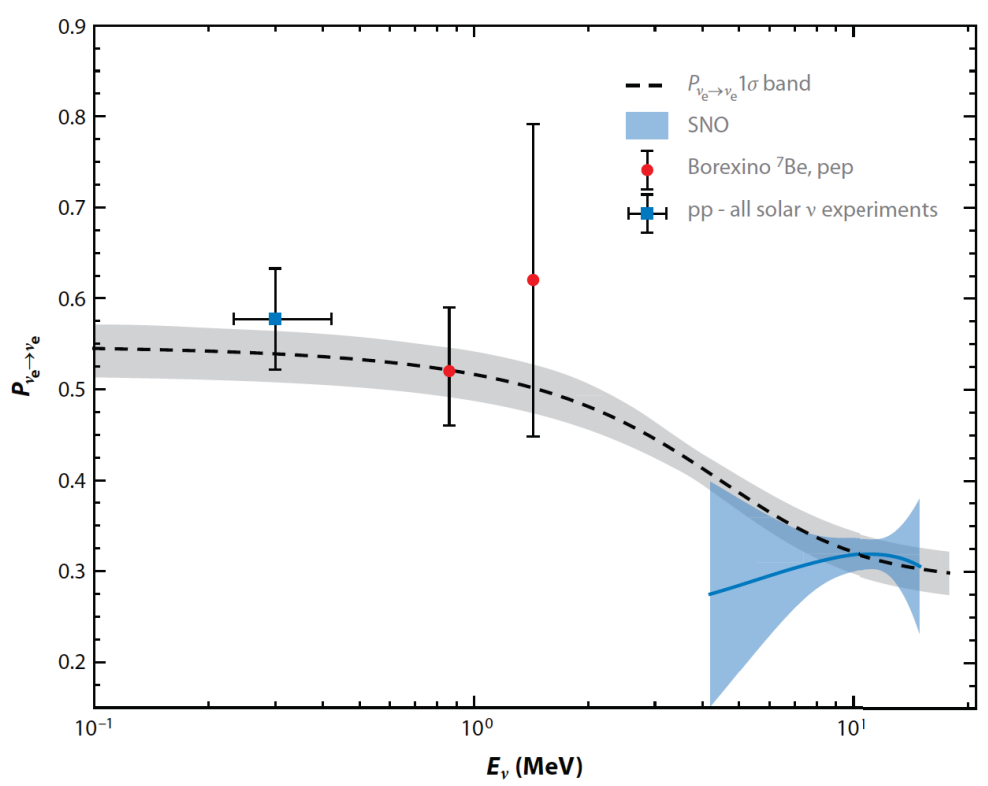

Figure 1. Theoretical expectation for the electron neutrino survival probability $\mathrm{P}_{e e}$ according to the LMA-MSW solution (dashed line, with grey band representing theoretical uncertainty) and current experimental knowledge from all solar neutrino experiment. The low energy point is calculated from gallium solar neutrino data using measured ${ }^{7} \mathrm{Be}$ data from Borexino. Figure taken from [15].

future prospectives, both of existing experiments and of new projects under construction or foreseen for the near future; finally, in section 6 I will discuss briefly the use of the Borexino detector as a tool to search for sterile neutrinos by means of strong neutrino and anti-neutrino artificial sources.

\section{$3{ }^{8} \mathrm{~B}$ neutrinos}

A very interesting feature of the MSW-LMA solution is the so called "up-turn", i.e. the rise of the $\mathrm{P}_{e e}$ right below the ${ }^{8} \mathrm{~B}$ solar neutrino energies. It is important for two main reasons: on one hand, it is the signature of the MSW effect, being the solar neutrino oscillations essentially vacuum oscillations below the critical energy $\mathrm{E}_{v} \approx 1.9 \mathrm{MeV}\left(\mathrm{P}_{e e} \simeq 0.57\right.$ for $\left.\mathrm{E}_{v} \ll 1.9 \mathrm{MeV}\right)$ and being dominated by matter effects at high energy $\left(\mathrm{P}_{e e} \simeq 0.31\right.$ for $\left.\mathrm{E}_{v} \gg 1.9 \mathrm{MeV}\right)$. The region around $1.9 \mathrm{MeV}$ is called the transition region and the rise of the $\mathrm{P}_{e e}$ below $\approx 3 \mathrm{MeV}$ the up-turn (see Fig. 1). On the other hand, significant deviations from the expected $\mathrm{P}_{e e}$ shape might provide hints of new physics effects, such as, e.g., non-standard neutrino interactions in the Sun or in the detector, or the existence of sterile neutrinos.

Four different experiments (SK, SNO, Borexino and KamLAND) using two different techniques have measured the solar neutrino flux in the range $3-10 \mathrm{MeV}$, where the spectrum is totally dominated by ${ }^{8} \mathrm{~B}$ neutrinos. Fig. 2 summarises the result of the experiments.

Borexino detects solar neutrinos by means of elastic scattering of electrons in a liquid scintillator and has been the first experiment capable of measuring ${ }^{8} \mathrm{~B}$ events with an electron recoil energy threshold at $3 \mathrm{MeV}$. The top-left plot in Fig. 2 shows the Borexino result, which is well consistent 
with LMA-MSW prediction, except perhaps for the lowest energy bin. KamLAND (top-right plot in Fig. 2), which uses the same technique with a higher energy threshold, finds a similar result, again with an apparent deficit in the lowest energy bin.

SNO has reduced the energy threshold with a dedicated analysis effort called LETA [18]. Its result (bottom-left plot in Fig. 2) is well consistent with LMA-MSW except two points at low energy, which yield a result lower than expected.

Super-kamiokaNDE measures the ${ }^{8} \mathrm{~B}$ neutrino flux by detecting Cherenkov light of scattered electrons. A major improvement of electronics and triggering capabilities (SK-IV phase, since 2008) has lowered the detection threshold of solar neutrinos down to $3.5 \mathrm{MeV}$. This fact has opened the possibility to study the "up-turn" region. The bottom-right plot in Fig. 2 (from [19]) shows that LMA-MSW solutions fits very well the energy distribution of the events, with a statistically not significant tension in the very low energy bins.

Although not statistically significant, all experiments find consistently lower signal in the low energy portion of the ${ }^{8} \mathrm{~B}$ solar neutrino spectrum, where the "up-turn" of the $\mathrm{P}_{e e}$ is expected. It is too early to say whether these are just statistical fluctuations, systematic errors close to the detection thresholds, or hints of new physics. We just underline that the latter possibility is of course very interesting and motivates the efforts of existing or future experiments. SK and Borexino will continue to take data for some years and will improve the result.

Recently, the SK collaboration has published a very interesting measurement of ${ }^{8} \mathrm{~B}$ neutrino flux day-night asymmetry [20]. At ${ }^{8} \mathrm{~B}$ energies the regeneration of the neutrinos during their path across the Earth is not negligible and the cited paper has provided the first evidence of that effect, although only at $2.7 \sigma$ level. If confirmed, this result would be the first direct detection of MSW effect of neutrinos in matter.

\section{4 sub-MeV solar neutrino detection with Borexino}

Borexino is the only experiment that has a sufficiently low detection threshold and a low enough intrinsic radioactive background to detect sub-MeV solar neutrinos. This section is therefore limited to Borexino results.

The main goal of the experiment was the detection of the monochromatic neutrinos that are emitted in the electron capture of ${ }^{7} \mathrm{Be}$ in the Sun with 5\% precision [21][22]. In order to achieve such a goal the intrinsic contamination of the liquid scintillator and the external radiation must be reduced to unprecedented low levels. In particular, design goals of Borexino required ${ }^{238} \mathrm{U}$ and ${ }^{232} \mathrm{Th}$ contents below $10^{-16} \mathrm{~g} / \mathrm{g},{ }^{40} \mathrm{~K}_{\text {nat }}$ content below $10^{-14} \mathrm{~g} / \mathrm{g}$ and a few counts per day maximum in the fiducial volume from ${ }^{85} \mathrm{Kr}$ and ${ }^{39} \mathrm{Ar}$. These goals, thanks to a long and dedicated effort and the development of innovative purification techniques, have all been met and in some cases by far exceeded, as e.g. for ${ }^{238} \mathrm{U}$ and ${ }^{232} \mathrm{Th}$ contents, which are both below $10^{-18} \mathrm{~g} / \mathrm{g}$. See [23], [24], [25], [32] and references therein for more details.

The precision measurement of ${ }^{7} \mathrm{Be}$ was obtained during the first stage of the experiment [27][28]. Real time detection of the events and detector stability allowed to study the day-night effect of the ${ }^{7} \mathrm{Be}$ solar neutrino signal, which yielded the exclusion of the LOW solution of the neutrino oscillation based on solar data alone [29]. The very low background of the detector, a refined analysis on threefold coincidences and an innovative discrimination method of $\beta^{+}$events based on the positronium formation, made it possible to explore the $1-2 \mathrm{MeV}$ region with unprecedented sensitivity. This led to the first observation of solar neutrinos from the basic pep reaction [30] and to the best upper limit for the CNO production in the Sun. 

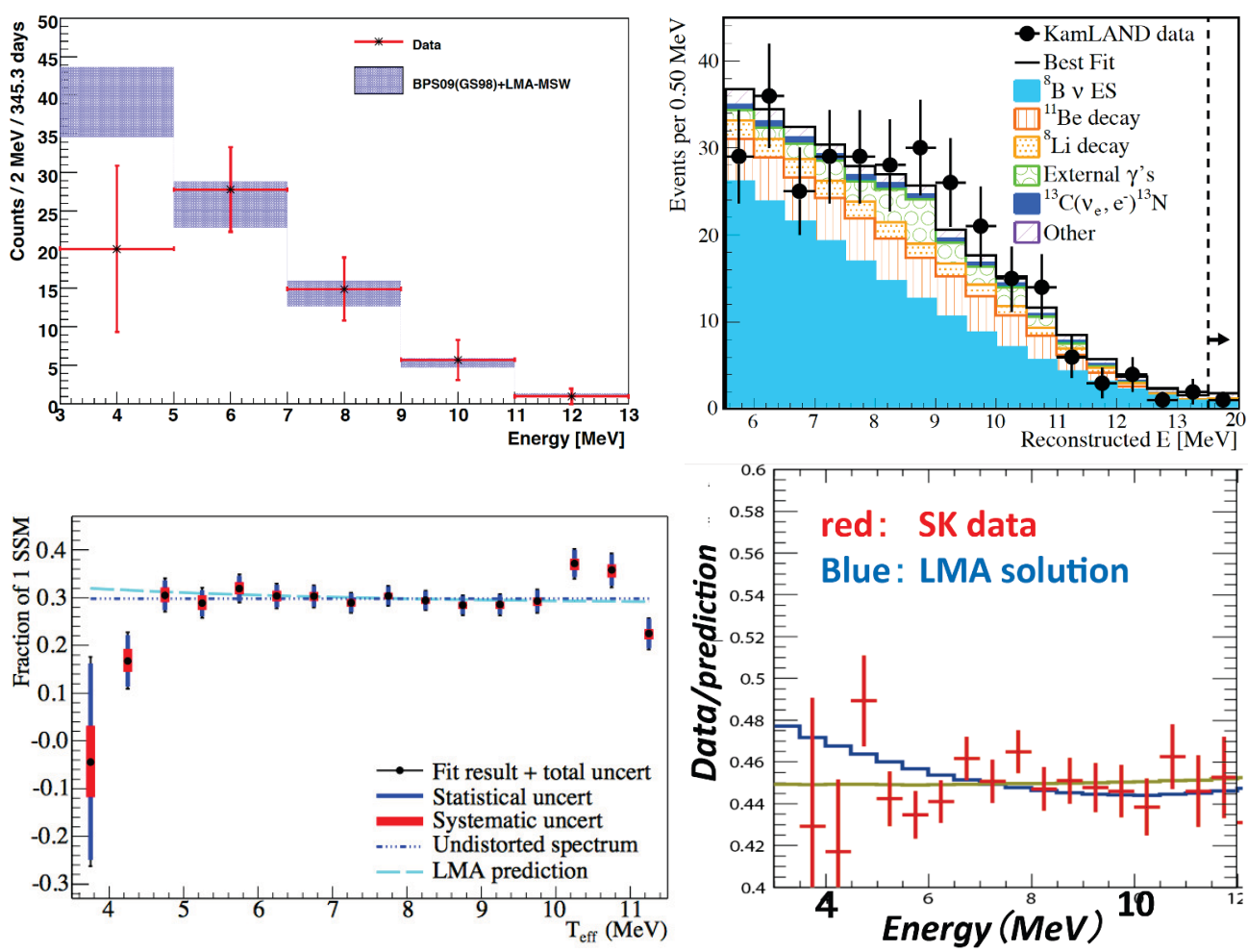

Figure 2. Results for the search of the ${ }^{8} \mathrm{~B}$ up-turn from four experiments: Top Left) Borexino [16]; Top Right) KamLAND [17]; Bottom Left) SNO [18]; Bottom Right) Super-kamiokaNDE [19]. Although not statistically significant, all experiments find consistently lower signal in the low energy portion of the ${ }^{8} \mathrm{~B}$ solar neutrino spectrum, where the "up-turn" of the $\mathrm{P}_{e e}$ is expected.

Very recently (after the presentation in Crete) Borexino has also completed and published the first direct real time measurement of pp neutrinos. Details can be found in ??. A complete account of Borexino results can also be found in [32].

Fig. 1 summarises well the impact of Borexino measurements on our current knowledge of $\mathrm{P}_{e e}$ at the time of the conference. An update of this figure can be found in ??. A direct measurement of CNO neutrinos is also among the goals of the experiment, although detector stability limitations might prevent a reliable measurement.

\section{Future perspectives}

It is not possible, within the allowed space of this paper, to give a comprehensive review of all proposed projects on solar neutrinos. I will limit the discussion for those who are already approved or very mature for approval.

Among the running experiments, Borexino and Super-kamiokaNDE are the ones that could yield the most interesting results. 
After a successful purification campaign, the Borexino experiment has reduced the scintillator background even further with respect to 2007-2010 data. In particular, the ${ }^{85}$ background was significantly reduced, opening the way to a first direct measurement of pp neutrinos. Borexino might possibly be able to put a strong constraint on CNO solar neutrinos, which would be very useful to solve the metallicity saga. However, besides a strong reduction of ${ }^{210} \mathrm{Bi}$ background, which was partially achieved by means of purifications, a much higher detector stability is required to be able to measure such a low signal (about 3 times smaller than the already difficult pep signal). Further purification campaigns might be attempted 2015, possibly with an improved water plant. If successful, a lower ${ }^{210} \mathrm{Bi}$ background united with an improved detector stability might open a window of opportunity for CNO neutrinos. Se son rose, fioriranno.

The most important future project in solar neutrino physics, besides those already running, is $\mathrm{SNO}+[33]$.

The $\mathrm{SNO}+$ collaboration is constructing a liquid scintillator experiment re-using the vessel and the photomultipliers of the SNO experiment. The scintillator is linear alkylbenzene (LAB) doped of PPO, which is safe (high flash point), low cost, and compatible with the existing acrylic vessel. The project has two main goals: the study of pep and CNO neutrinos, and the search of neutrino less double beta decay with Te dissolved in the scintillator.

As far as solar neutrinos are concerned, the higher depth of the Sudbury mine compared to Gran Sasso and Kamioka yields a much lower cosmogenic background from ${ }^{11} \mathrm{C}$ (700 times less than Kamioka, 100 times less than LNGS). According to the simulations, $\mathrm{SNO}+$ may be able to measure the CNO neutrino flux with $6 \%$ error. This precision is sufficient to measure the metal content of the solar core in a model independent way and possibly solve the solar metallicity problem. The expected signal in SNO+ for pep and CNO neutrinos is shown in Fig. 3. This sensitivity, however, will be possible only the the background induced by ${ }^{210} \mathrm{Bi}$ is at least as low as the Borexino one, or maybe better.

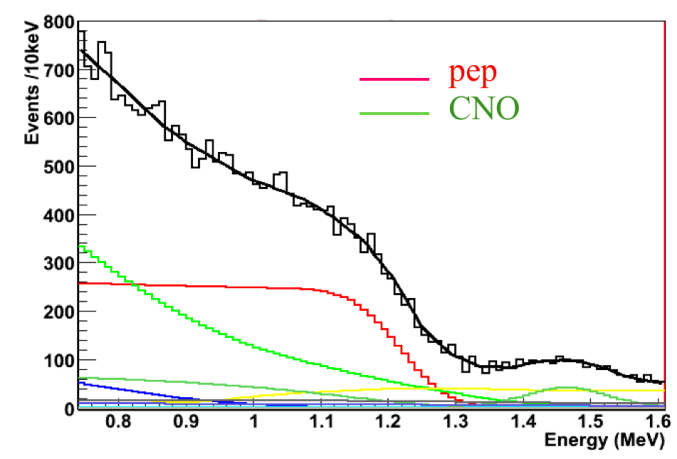

Figure 3. The signal expected in $\mathrm{SNO}+$ in three years of data taking for $\mathrm{CNO}$ and pep neutrinos. The low cosmogenic ${ }^{11} \mathrm{C}$ background and the high mass make $\mathrm{SNO}+$ may allow a $6 \%$ measurement of $\mathrm{CNO}$ neutrinos in three years of data.

Another more ambitious project for solar neutrino physics is LENS, Low Energy Neutrino Spectroscopy. This experiment aims at the precise measurement of all solar neutrino components by detecting neutrinos via charged current interactions (inverse beta decay) with ${ }^{115} \mathrm{In}$. 

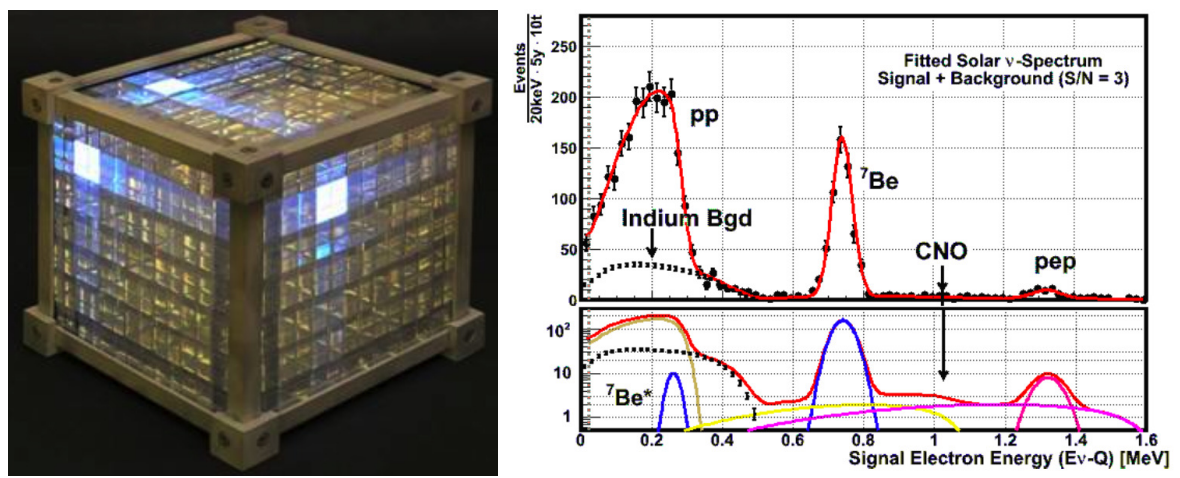

Figure 4. Left) Lens conceptual design. Isotropically emitted scintillation light is channeled to the outside of the detector along the main axes via total internal reflection Right) Lens expected spectral resolution on solar neutrinos. If the detector will work as anticipated, LENS will give a beautiful spectral measurement of all solar neutrino components separately, with very high precision and low background.

The experimental tool used in the LENS detector for the detection of solar neutrinos is the tagged capture of $v_{e}$ s on ${ }^{115}$ In via charged current inverse beta decay. The tagged technique has two outstanding advantages over competing experiments: first, there is a one-to-one correspondence between the incoming neutrino energy and the measurable electron energy $\mathrm{E}_{v}=\mathrm{E}_{e}+\mathrm{Q}_{d}\left(\mathrm{Q}_{d}\right.$ : capture threshold), and second, the $\gamma$-cascade allows the application of time/space coincidence techniques to suppress ubiquitous radioactive backgrounds as well as the inherent background from the beta decay of ${ }^{115} \mathrm{In}$.

A modular detector is required. The LENS detector is a novel Scintillation Lattice Chamber, an optically segmented, three dimensional array of 0.51 cells of liquid scintillator loaded with $8-10 \mathrm{wt} \%$ Indium. The scintillation signal from each cell will be always viewed by the same set of 3-6 phototubes. Thus, the full scale LENS of 125 tons InLS, though large in size, is in essence, a large array of small detectors capable of bench-top precision nuclear spectroscopy. It will provide extraordinary spatial resolution in a large mass of liquid scintillator through segmentation rather then time of flight information, which allows adequate background rejection using the time/space coincidence tag even for low energy ( $100 \mathrm{keV}$ ) events.

If successful, this experiment might really represent the future of solar neutrino physics because of the superbe energy resolution possible for all neutrino energies. A spectrum of the expected signal in LENS is shown in Fig. 4 together with a conceptual design of the detector.

\section{The Borexino detector as a tool to search for sterile neutrinos}

The record radio purity of the scintillator volume, its relatively large size and its capability to detect and locate both neutrino and anti-neutrino events, make Borexino an ideal instrument for the study of short distance neutrino oscillations by means of intense neutrino sources located close to or, maybe in the future, inside the detector.

The SOX project [39] aims at the search of sterile neutrinos or the study of other non-standard short distance effects using an intense 5-10 MCi neutrino source made with about $35 \mathrm{~kg}$ of ${ }^{51} \mathrm{Cr}$ activated in a nuclear reactor and with a $100-150 \mathrm{kCi}$ anti-neutrino source made of ${ }^{144} \mathrm{Ce}$ [38]. The experiment will probe the so called reactor and gallium anomalies and search for sterile neutrinos 
with high sensitivity. Fig. 5 shows the sensitivity of the experiment both to neutrino (left plot) and anti-neutrino sources (right plot). The relatively large size of the Borexino fiducial volume (about $6 \mathrm{~m}$ diameter for neutrino events, about $8 \mathrm{~m}$ for anti-neutrino events) allow to detect directly oscillations waves if the $\delta m_{s}^{2}$ with the sterile component is of the order of $1 \mathrm{eV}^{2}$. This makes SOX a rather unique experiment. The existing plan is to perform first the measurement with anti-neutrino source (possibly starting in late 2015 or early 2016 and taking data for about 18 months) and then perform the measurement with the ${ }^{51} \mathrm{Cr}$ neutrino source. Besides sterile neutrinos, the combined measurement of neutrinos and anti-neutrinos yield valuable information also on the Weinberg angle and on nonstandard neutrino interactions at low energy. See [39] for more details.

\section{Acknowledgements}

I wish to thank the Laboratori Nazionali del Gran Sasso for their continual support. My special thanks also to the organisers of this conference for the kind and efficient hospitality. SOX is funded by ERC-2012-AdG project N. 320873 (FP7), P.I Marco Pallavicini.

\section{References}

[1] B. T. Cleveland et al., Astroph. J. , 496 (1998) 505-526

[2] K.S. Hirata et al. (KamiokaNDE-II collaboration), Phys. Rev. Lett 63 (1989) 16-19

[3] Y. Fukuda et al. (KamiokaNDE-III collaboration) Phys. Rev. Lett. 77 (1996) 1683-1686

[4] S. Fukuda et al. (Super-KamiokaNDE-I collaboration) Phys. Lett. B539 (2002) 179-187

[5] P. Anselmann et al. (Gallex collaboration) Phys. Lett. B285 (1992) 376-389

[6] J. N. Abdurashitov et al. (SAGE collaboration), Phys. Lett. B328 (1994) 234-248

[7] Q. R. Ahmad et al. (SNO collaboration), Phys. Rev. Lett. 87 (2001) 071301

[8] Q. R. Ahmad et al. (SNO collaboration), Phys. Rev. Lett. 89 (2002) 011301

[9] Y. Fukuda et al. (Super-KamiokaNDE-I collaboration) Phys. Rev. Lett. 81 (1998) 1562-1567;
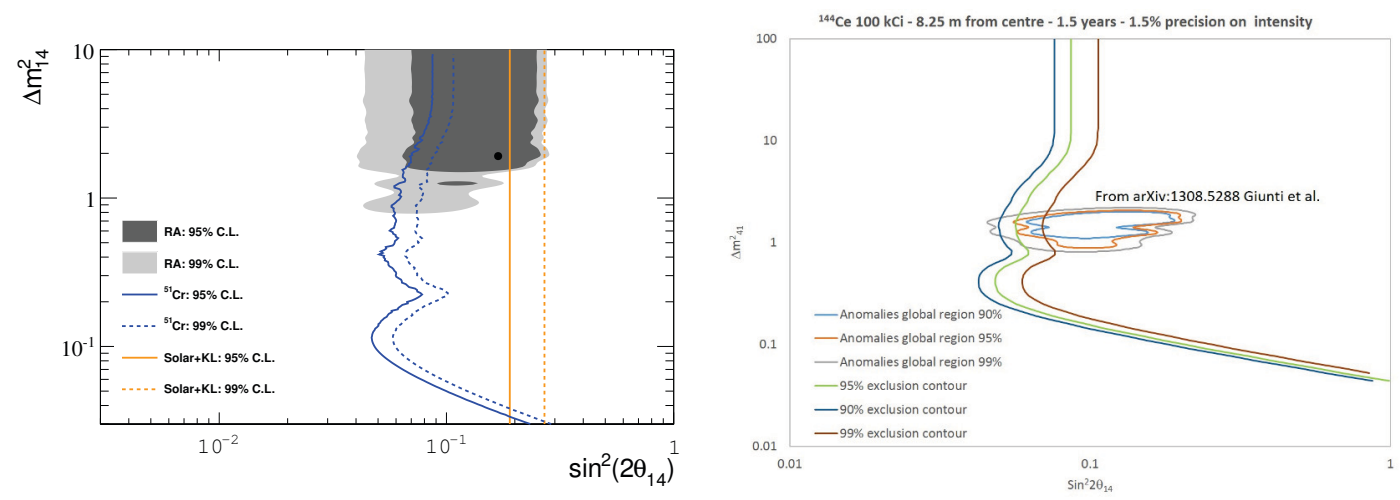

Figure 5. Left ${ }^{51} \mathrm{Cr}$ SOX sensitivity compared with the allowed reactor anomaly region. The yellow line is the limit from solar neutrino analysis. Right) ${ }^{144} \mathrm{Ce}$ SOX sensitivity compared with the region obtained as global fit from all neutrino anomalies [37]. 
[10] L. Wolfenstein, Phys. Rev. D17 (1978) 2369

[11] L. Wolfenstein, Phys. Rev. D20 (1978) 2634

[12] S.P. Mikheyev and A.Y. Smirnov Sov. J. Nucl. Phys. 42 (1985) 1913

[13] S.P. Mikheyev and A.Y. Smirnov Nuovo Cim. 9C (1986) 17

[14] K. Eguchi et al. (KamLAND collaboration) Phys. Rev. Lett. 90 (2003) 021802

[15] W.C. Haxton, R.G. Hamish Robertson and A.M. Serenelli, Annu. Rev. Astron. Astrophys. 51 (2013) 21-61

[16] G. Bellini et al. (Borexino Collaboration) Phys. Rev. D82 (2010) 033006

[17] B. Aharmim et al. (KamLAND Collaboration) Phys. Rev. C84 (2011) 035804

[18] B. Aharmim et al. (SNO Collaboration) Phys. Rev. C81 (2010) 055504

[19] Y. Suzuki (for the Super-kamiokaNDE Collaboration) Neutrino Telescopes (2013)

[20] A. Renshaw et al. (Super-kamiokaNDE collaboration ) Phys. Rev. Lett. 112 (2014) 091805

[21] G. Alimonti et al. (Borexino Collaboration), Astropart. Phys. 16 (2002) 205

[22] C. Arpesella et al. (Borexino Collaboration), Astropart. Phys. 18 (2002) 1

[23] H. O. Back et al. (Borexino Collaboration), Nucl. Instrum. Methods A 609 (2009) 58

[24] H. O. Back et al. (Borexino Collaboration), Phys. Lett. B 563 (2003) 23

[25] H. O. Back et al. (Borexino Collaboration), Phys. Lett. B 525 (2002) 29

[26] G. Bellini et al. (Borexino Collaboration) arXiV 1308.0443v1, submitted to Phys. Rev. D

[27] C. Arpesella et al. (Borexino Collaboration), Phys. Lett. B 658 (2008) 101

[28] G. Bellini et al. (Borexino Collaboration) Phys. Rev. Lett. 107 (2011) 141302

[29] G. Bellini et al. (Borexino Collaboration) Phys. Lett. B707 (2012) 22

[30] G. Bellini et al. (Borexino Collaboration) Phys. Rev. Lett. 108 (2012) 051302

[31] G. Bellini et al. (Borexino Collaboration) Nature 512 (2014) 383

[32] G. Bellini et al. (Borexino Collaboration) Phys. Rev. D89 (2014) 112007

[33] N. Tolich for the SNO+Collaboration NNN 09, http://nnn09.colostate.edu/Talks/Session05/

[34] C. Grieb, Nucl. Phys. B 168 (2007) 122

[35] M. Cribier et al., Phys. Rev. Lett. 107 (2011) 201801

[36] G. Bellini et al., (Borexino Collaboration) JHEP 08 (2013) 038

[37] C. Giunti et al. Phys. Rev. D88 (2013) 073008

[38] M. Cribier et al., Phys. Rev. Lett. 107 (2011) 201801

[39] G. Bellini et al. (SOX collaboration) JHEP 08 (2013) 038 